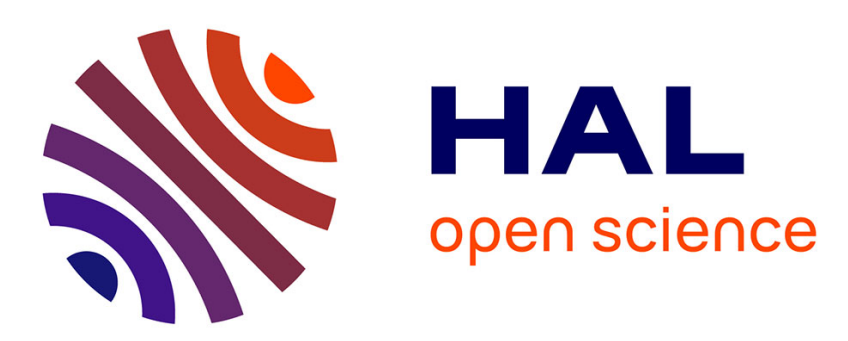

\title{
A Sharp Sufficient Condition for B-Spline Vector Field Invertibility. Application to Diffeomorphic Registration and Interslice Interpolation.
}

\author{
Michaël Sdika
}

\section{- To cite this version:}

Michaël Sdika. A Sharp Sufficient Condition for B-Spline Vector Field Invertibility. Application to Diffeomorphic Registration and Interslice Interpolation.. SIAM Journal on Imaging Sciences, 2013, 6 (4), pp.2236-2257. 10.1137/120879920 . hal-01902498

\section{HAL Id: hal-01902498 \\ https://hal.science/hal-01902498}

Submitted on 11 Jan 2019

HAL is a multi-disciplinary open access archive for the deposit and dissemination of scientific research documents, whether they are published or not. The documents may come from teaching and research institutions in France or abroad, or from public or private research centers.
L'archive ouverte pluridisciplinaire HAL, est destinée au dépôt et à la diffusion de documents scientifiques de niveau recherche, publiés ou non, émanant des établissements d'enseignement et de recherche français ou étrangers, des laboratoires publics ou privés. 


\title{
A Sharp Sufficient Condition for B-Spline Vector Field Invertibility. Application to Diffeomorphic Registration and Interslice Interpolation*
}

\author{
Michaël Sdika ${ }^{\dagger}$
}

\begin{abstract}
In this paper, a new sufficient condition for the invertibility of a B-spline vector field as well as its extension to spatio-temporal vector fields are presented. The new condition, while guaranteeing that the vector field is diffeomorphic, is sharp enough to allow large deformations. The complexity of the constraint is also discussed. A new diffeomorphic registration algorithm using this condition and its application to interslice interpolation are presented and evaluated on synthetic images and in vivo magnetic resonance $T_{2}$ images of the brain. The registration method has also been compared to some state-of-the-art registration methods.
\end{abstract}

Key words. diffeomorphism, image registration, B-spline, interslice interpolation

AMS subject classifications. 65D07, 65D15, 57R25, 57R50, 68U10, 90C30, 90C90

DOI. $10.1137 / 120879920$

1. Introduction. Image registration is the process of finding, given two images, a transformation that maps the pixels of one image (the reference image) to the corresponding pixels in the other image (the floating image). Since its introduction as an image processing tool in [12], it is now a common tool for imaging based studies and has been extensively used for atlas based segmentation [51], [44], [32]; morphometry study [16], [5]; motion estimation [42]; slice interpolation [35]; and handwritten character recognition [10], [50].

The mapping from the reference to the floating image to be estimated is a geometric transformation. It can be a simple translation or an isometry for the registration of a rigid body in a different position. In the general case, it is a nonlinear mapping, represented by a vector field.

A common requirement for the transformation to estimate is, besides its smoothness, to be invertible. A diffeomorphic mapping is indeed physically meaningful when this mapping represents the deformation of the same object at several timepoints, for example, when studying the deformation of the cardiac muscle [37] or the lung [48]. When the objects to register are different, invertibility may be debatable but is still commonly used to preserve the topology of the object in the floating image after deformation. Diffeomorphism may also be necessary to register vector or tensor images, for example, diffusion tensor images. In this case, nonsingularity in the Jacobian map is necessary to correctly orient the tensor or vector pixels (see for example [13]). Diffeomorphic registration can also be necessary if the inverse of the transformation is concretely needed for further processing as for example in [39], in which the

\footnotetext{
* Received by the editors June 6, 2012; accepted for publication (in revised form) August 12, 2013; published electronically November 19, 2013.

http://www.siam.org/journals/siims/6-4/87992.html

†Université de Lyon, CREATIS; CNRS UMR5220; Inserm U1044; INSA-Lyon; Université Lyon 1, France (michael. sdika@creatis.insa-lyon.fr).
} 
inverse is needed to compute the accuracy map of a segmentation atlas. Finally, diffeomorphic transformation can also be useful for parameterization of a three-dimensional (3D) surface. In this case, one looks for a mapping with specific properties (for example, area or angular preservation) from a two-dimensional (2D) region to a 3D surface [22], [27], [7]. This mapping should obviously be invertible.

In the literature of nonrigid registration, two models can be distinguished to represent the transformation: either a closed form expression parameterized by a finite-dimensional vector or a dense array of vectors giving the position of each pixel in the other image. In the former case, the vector field is often a linear combination of vector basis functions such as B-splines.

Dense diffeomorphic registration have been extensively studied; see [45], [9], [8]. The main idea is to model transformations as solutions at $t=1$ of equations $\frac{\partial T}{\partial t}=v(t, T)$, where the velocity $v$ is now the unknown of the problem. This requires the addition of the time dimension to the unknown and the resolution of a differential equation to compute the transformation. Fast registration can, however, be achieved when the velocity is stationary. In this case the exponential map, which maps the velocity to the transformation, can be computed efficiently as in [4], [25], or [49].

However, there are several advantages in using parametric models for the transformation: transformation resolution can be chosen independently from the image resolution, values of the transformation are continuously defined in the model, and derivatives can be computed exactly from analytical expressions. More specifically, an exact and consistent Jacobian can be computed for further analysis.

Dense and parametric registration can also be distinguished by the moment the approximation occurs in the resolution process. In dense diffeomorphic registration, the theory is developed for continuous transformation; the solution is then approximated by discretization of the transformations and equations from the theory. With parametric diffeomorphic registration, the approximation comes first by restricting the search space of the solution to some set of parameterized transformations. This will guarantee that theoretical properties derived for these transformations will apply to the objects effectively used in the software implementation. The difficulty, however, is to be able to have a sufficiently large search space to model the transformation of interest.

When the deformation is modeled as a B-spline vector field, diffeomorphic registration is based on solving the registration problem with the additional constraint that the transformation Jacobian be (continuously) positive.

In [38], pixelwise constraints are included in the registration framework. The Jacobian of the transformation is guaranteed to be positive on pixels, and its negativity can be further penalized between pixels. However, if nondiffeomorphic transformations are strongly penalized, invertibility is not strictly guaranteed.

In [33], the optimization is performed one node at a time. For 2D registration and when the transformation is modeled with degree-1 B-splines, this allows us to reduce the diffeomorphic constraint to two linear inequality constraints per node. This approach has been extended in [34] to 3D registration with the use of interval arithmetic to solve the constraints, but it is still restrained to degree-1 B-splines.

As the transformation is continuously defined by a finite number of parameters (the Bspline coefficients), it would be interesting to transfer the infinite number of constraints (the 
continuous positivity of the Jacobian) to the parameter space. To the best of our knowledge, two sufficient conditions have been proposed to guarantee the invertibility of the transformation.

The first condition, proposed in [14], is a bound constraint on the coefficients of the displacement field. It has been used in the context of image registration in [36]. However, as the constraint is very restrictive, the transformation is modeled as a composition of B-spline vector fields, losing the advantages of a parametric representation.

A bound on the finite differences of the displacement field coefficients has been proposed in [28] and extended in [15]. This constraint is much less restrictive than that of [14]: compositions are still necessary but in a much lower quantity. From a practical point of view, the authors propose not to enforce the constraint but to use it as a regularizer.

In this paper, a new sufficient condition for continuous positivity of the Jacobian of a $\mathrm{B}$-spline vector field is derived. This condition is much less restrictive than state-of-the-art sufficient conditions. No composition is needed to obtain a correct diffeomorphic registration of common images. The condition is used as a hard constraint to develop a new diffeomorphic registration algorithm, and its use in the context of interslice interpolation is discussed.

B-spline vector fields are introduced in section 2, and the condition for vector fields and spatio-temporal vector fields is proposed in section 3. Analysis of the complexity and the sharpness of the proposed conditions is given in sections 4 and 5 . In section 6 , the condition is used to build a diffeomorphic registration algorithm, and the spatio-temporal version of the condition is used to propose a well-defined interslice interpolation method. Numerical experiments on synthetic and in vivo images and comparisons to some state-of-the-art algorithms are presented in section 7 .

2. B-spline vector field. The $D$-dimensional vector field is defined using B-splines of degree $n$ :

$$
\begin{aligned}
T(x) & =x+\sum_{i} \tilde{c}_{i} \beta(x / h-i) \\
& =\sum_{i} c_{i} \beta(x / h-i),
\end{aligned}
$$

where $x$ and $c_{i}$ are in $\mathbb{R}^{D}, h \in \mathbb{N}^{D}$, and $i \in \mathbb{Z}^{D}$. The $\tilde{c}_{i}$ coefficients are used to model the displacement, and the transformation itself can be represented by the coefficients $c_{i}=\tilde{c}_{i}+i h$. Note that the two representations are equal only when the degree $n$ is greater than one. In this presentation $\beta$ will represent either the B-spline function of degree $n$ or its tensor product, depending on the context: $\beta(x)=\prod_{i=1}^{D} \beta\left(x_{i}\right)$. The definition and main properties of B-splines are given in Appendix A. The vector $h$ is the node spacing and will be considered as an integer for the sake of efficiency. This parameter allows us to choose the transformation resolution independently from the image resolution.

The exact computation of the transformation derivatives is made possible by the use of a continuous model. The B-spline representation allows further development, and, using property 5 of Appendix A, partial derivatives of the transformation can themselves be written with B-splines (see [47]):

$$
\frac{\partial T}{\partial x_{l}}(x)=\sum_{i} d_{i}^{l} \tilde{\beta}^{l}(x / h-i)
$$


The $\left(d_{i}^{l}\right)_{i}$ are the finite difference of the B-spline coefficient in the $x_{l}$ direction:

$$
\begin{aligned}
d_{i}^{l} & =\frac{\tilde{c}_{i}-\tilde{c}_{i-e_{l}}}{h_{l}}+e_{l} \\
& =\frac{c_{i}-c_{i-e_{l}}}{h_{l}},
\end{aligned}
$$

where $e_{l}$ is the $l$ th vector of the canonical basis and $\tilde{\beta}^{l}$ is the tensor product of $\beta^{-}$, the B-spline of degree $n-1$, and $\beta$ :

$$
\tilde{\beta}^{l}(x)=\beta^{-}\left(x_{l}+\frac{1}{2}\right) \prod_{k \neq l} \beta\left(x_{k}\right) .
$$

From the expression (2.1) of the derivatives of $T$ and using the multilinearity of the determinant, a B-spline representation of the Jacobian $J$ of the transformation can be derived as follows:

$$
\begin{aligned}
J(x) & =\operatorname{det}\left(T^{\prime}(x)\right) \\
& =\operatorname{det}\left(\frac{\partial T}{\partial x_{1}}(x), \ldots, \frac{\partial T}{\partial x_{D}}(x)\right) \\
& =\operatorname{det}\left(\sum_{i_{1}} d_{i_{1}}^{1} \tilde{\beta}_{i_{1}}^{1}(x), \ldots, \sum_{i_{D}} d_{i_{D}}^{D} \tilde{\beta}_{i_{D}}^{D}(x)\right) \\
& =\sum_{i_{1}, \ldots, i_{D}} \underbrace{\operatorname{det}\left(d_{i_{1}}^{1}, \ldots, d_{i_{D}}^{D}\right)}_{J_{i_{1}, \ldots, i_{D}}} \underbrace{\tilde{\beta}_{i_{1}}^{1}(x) \ldots \tilde{\beta}_{i_{D}}^{D}(x)}_{\tilde{\beta}_{i_{1}, \ldots, i_{D}}(x)},
\end{aligned}
$$

where $\tilde{\beta}_{i}^{l}(x)=\tilde{\beta}^{l}(x / h-i)$. The Jacobian of $T$ can be expressed as the linear combination of the $J_{i_{1}, \ldots, i_{D}}$, the B-spline coefficient "Jacobians," weighted by $\tilde{\beta}_{i_{1}, \ldots, i_{D}}$, a product of tensor Bsplines. This formula is very inefficient if used to compute $J(x)$ but is theoretically important for the derivation of our condition.

\section{A sufficient condition for B-spline vector field invertibility.}

3.1. D-dimensional B-spline vector field. As in [14] and [15], our invertibility condition is found by bounding the Jacobian on $\mathbb{R}^{D}$. The sufficient invertibility condition is then that the lower bound must remain positive. To find the condition, the following definition is needed.

Definition 3.1. $E_{D}$ is the set of active D-tuples in the Jacobian of a D-dimensional B-spline vector field of degree $n$ :

$$
E_{D}=\left\{i_{1}, \ldots, i_{D} / \exists x \tilde{\beta}_{i_{1}, \ldots, i_{D}}(x) \neq 0\right\}
$$

In other words, $E_{D}$ is the set of multi-indexes that are actually used in the Jacobian computation. The sum in the expression (2.2) can therefore be restricted to the indices of $E_{D}$. In the following proposition, $E_{D}$ is characterized.

Proposition 3.2. Let $E_{D}$ be the set of active D-tuples in Jacobians of D-dimensional Bspline vector fields of degree $n$, and let $I^{-}=[-n, n-1]$ and $I=[-n, n]$; then $E_{D}$ is the set 
of index D-tuples $i_{1}, \ldots, i_{D}$ such that for all $a \neq b$,

$$
i_{b}-i_{a} \in \prod_{k} I_{k},
$$

where

$$
I_{k}=\mid \begin{array}{cc}
I^{-} & \text {if } k=a, \\
-I^{-} & \text {if } k=b, \\
I & \text { otherwise. }
\end{array}
$$

Proof. Let us first define $E_{D}(x)$, the set of indices having an influence in the computation of Jacobian functions at $x$. Looking at (2.2), this set can be expressed as

$$
E_{D}(x)=\left\{i_{1}, \ldots, i_{D} / \tilde{\beta}_{i_{1}, \ldots, i_{D}}(x) \neq 0\right\} .
$$

Now let's try to give an explicit expression of $E_{D}$, the union over $x$ of these sets. If $i_{1}, \ldots, i_{D} \in$ $E_{D}$, then $\exists x$ such that $\tilde{\beta}_{i_{1}, \ldots, i_{D}}(x)$ is not null. Given that

$$
\begin{aligned}
\tilde{\beta}_{i_{1}, \ldots, i_{D}}(x) & =\prod_{l} \tilde{\beta}_{i_{l}}^{l}(x) \\
& =\prod_{l} \beta^{-}\left(\frac{x_{l}}{h_{l}}-i_{l}^{l}+\frac{1}{2}\right) \prod_{k \neq l} \beta\left(\frac{x_{k}}{h_{k}}-i_{l}^{k}\right),
\end{aligned}
$$

then, for each $l$, the following expression is not null:

$$
\beta^{-}\left(\frac{x_{l}}{h_{l}}-i_{l}^{l}+\frac{1}{2}\right) \prod_{k \neq l} \beta\left(\frac{x_{l}}{h_{l}}-i_{k}^{l}\right) .
$$

Using Lemma B.1 of Appendix B, for each $j \neq l$ and $k \neq l$,

$$
i_{j}^{l}-i_{k}^{l} \in I \text {. }
$$

Using Lemma B.2, for each $k \neq l$,

$$
i_{k}^{l}-i_{l}^{l} \in I^{-} .
$$

Consequently, for $a \neq b$, we have

$$
i_{b}^{k}-i_{a}^{k} \in \mid \begin{array}{cr}
I^{-} & \text {if } a=k, \\
-I^{-} & \text {if } b=k, \\
I & \text { otherwise. }
\end{array}
$$

Now that the Jacobian has been expanded with B-splines (expression (2.2)) and the active coefficients in this expansion have been identified (Proposition 3.2), the Jacobian can be bounded by its B-spline expansion coefficients using the bounding property of B-splines (property 6 of Appendix A). In the following theorem, upper and lower bounds on the Jacobian are derived for D-dimensional B-spline vector fields.

Theorem 3.3. Let $T$ be a D-dimensional B-spline of degree $n$ vector field, and let $J$ be its Jacobian; then, for all $x$, the following expression holds:

$$
\min _{i_{1}, \ldots, i_{D} \in E_{D}} J_{i_{1}, \ldots, i_{D}} \leq J(x) \leq \max _{i_{1}, \ldots, i_{D} \in E_{D}} J_{i_{1}, \ldots, i_{D}} .
$$


Proof. Let us first define the extrema over $E_{D}$ of the B-spline coefficients Jacobian:

$$
m=\min _{i_{1}, \ldots, i_{D} \in E_{D}} J_{i_{1}, \ldots, i_{D}}
$$

and

$$
M=\max _{i_{1}, \ldots, i_{D} \in E_{D}} J_{i_{1}, \ldots, i_{D}}
$$

So, for $i_{1}, \ldots, i_{D} \in E_{D}$, the following relation holds:

$$
m \leq J_{i_{1}, \ldots, i_{D}} \leq M
$$

For a given $x$, if this relation is multiplied by the positive tensor B-spline $\tilde{\beta}_{i_{1}, \ldots, i_{D}}(x)$ and if we consider the definition of $E_{D}$, then for all $i_{1}, \ldots, i_{D}$,

$$
m \tilde{\beta}_{i_{1}, \ldots, i_{D}}(x) \leq J_{i_{1}, \ldots, i_{D}} \tilde{\beta}_{i_{1}, \ldots, i_{D}}(x) \leq M \tilde{\beta}_{i_{1}, \ldots, i_{D}}(x) .
$$

Summing over $i_{1}, \ldots, i_{D}$ and using the partition of unity property of B-splines (property 4 of Appendix A), the following relation is obtained:

$$
m \leq J(x) \leq M
$$

This theorem gives upper and lower bounds of the continuous Jacobian of the transformation using only the B-spline coefficients. The Jacobian of a B-spline vector field is bounded by the extrema over $E_{D}$ of the B-spline coefficient "Jacobians." Regarding invertibility, the property one tries to have in the continuous domain (positivity of the Jacobian in $\mathbb{R}^{D}$ ) is transferred in the B-spline coefficient domain (positivity of all the B-spline coefficient Jacobians in $\left.E_{D}\right)$

For the sake of concreteness, the Jacobian bounds are given for low-dimensional problems. In one dimension, the following theorem is trivial.

Theorem 3.4. Let $T$ be a one-dimensional (1D) B-spline of degree $n$; then, for all $x$,

$$
\min _{i} d_{i} \leq T^{\prime}(x) \leq \max _{i} d_{i}
$$

In one dimension, the relationship between the continuous function and the B-spline coefficients is visible. Regarding invertibility, the strict monotony condition of the continuous function is transformed in the strict monotony of the B-spline coefficients.

In two dimensions, the set of active indices is

$$
E_{2}=\left\{(i, j) / j-i \in I^{-} \times\left(-I^{-}\right)\right\} .
$$

In three dimensions, $E_{3}$ is the set of triples $(i, j, k)$ such that

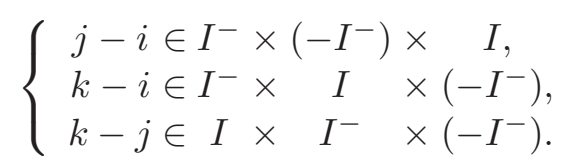


3.2. D-dimensional $+t$ B-spline vector fields. When studying the deformation of an object over a whole sequence of images at several timepoints, the motion can be advantageously represented by a continuous family of diffeomorphisms: for all $t, T(., t)$ should be a diffeomorphism. B-splines can also be used for interpolation in the time dimension:

$$
\begin{aligned}
T(x, t) & =x+\sum_{i, j} \tilde{c}_{i, j} \beta(x / h-i) \beta_{2}\left(t / h_{t}-j\right) \\
& =\sum_{i, j} c_{i, j} \beta(x / h-i) \beta_{2}\left(t / h_{t}-j\right),
\end{aligned}
$$

where $\beta_{2}$ is a B-spline function of degree $n_{2}$.

If the finite difference coefficients $d_{i, j}^{l}$ and the Jacobian coefficients $J_{i_{1}, \ldots, i_{D}, j_{1}, \ldots, j_{D}}$ are defined by

$$
d_{i, j}^{l}=\frac{c_{i, j}-c_{i-e_{l}, j}}{h_{l}}
$$

and

$$
J_{i_{1}, \ldots, i_{D}, j_{1}, \ldots, j_{D}}=\operatorname{det}\left(d_{i_{1}, j_{1}}^{1}, \ldots, d_{i_{D}, j_{D}}^{D}\right),
$$

then the Jacobian of $T(., t)$ can be bounded independently from $x$ and $t$ by the extrema of the Jacobian coefficients.

Theorem 3.5. Let $T$ be a continuous family of D-dimensional B-spline vector fields of degree $n$ in space and $n_{2}$ in time with $J$ its (spatial) Jacobian. Also, let $I_{2}=\left[-n_{2}, n_{2}\right]$ and $F_{D}=$ $\left\{j_{1}, \ldots, j_{D} / \forall k, \forall l, j_{k}-j_{l} \in I_{2}\right\}$; then, for all $x$ and $t$ the following expression holds:

$$
\min _{\substack{i_{1}, \ldots, i_{D} \in E_{D} \\ j_{1}, \ldots, j_{D} \in F_{D}}} J_{i_{1}, \ldots, i_{D}, j_{1}, \ldots, j_{D}} \leq J(x, t) \leq \max _{\substack{i_{1}, \ldots, i_{D} \in E_{D} \\ j_{1}, \ldots, j_{D} \in F_{D}}} J_{i_{1}, \ldots, i_{D}, j_{1}, \ldots, j_{D}}
$$

Proof. For a given $t, T(., t)$ is a B-spline vector field whose coefficients are

$$
c_{i}(t)=\sum_{j} c_{i, j} \beta_{2}\left(t / h_{t}-j\right),
$$

whose finite differences are

$$
\begin{aligned}
d_{i}^{l}(t) & =\frac{c_{i}(t)-c_{i-e_{l}}(t)}{h_{l}} \\
& =\sum_{j} \frac{c_{i, j}-c_{i-e_{l}, j}}{h_{l}} \beta_{2}\left(\frac{t}{h_{t}}-j\right) \\
& =\sum_{j} d_{i, j}^{l} \beta_{2}\left(\frac{t}{h_{t}}-j\right),
\end{aligned}
$$


and whose Jacobian coefficients at $t$ can be expressed as

$$
\begin{aligned}
J_{i_{1}, \ldots, i_{D}}(t) & =\operatorname{det}\left(d_{i_{1}}^{1}(t), \ldots, d_{i_{D}}^{D}(t)\right) \\
& =\operatorname{det}\left(\sum_{j_{1}} d_{i_{1}}^{1}(t) \beta_{2}\left(t / h_{t}-j_{1}\right), \ldots, \sum_{j_{D}} d_{i_{D}}^{D}(t) \beta_{2}\left(t / h_{t}-j_{D}\right)\right) \\
& =\sum_{j_{1}, \ldots, j_{D}} \operatorname{det}\left(d_{i_{1}, j_{1}}^{1}, \ldots, d_{i_{D}, j_{D}}^{D}\right) \prod_{l} \beta_{2}\left(t / h_{t}-j_{l}\right) \\
& =\sum_{j_{1}, \ldots, j_{D}} J_{i_{1}, \ldots, i_{D}, j_{1}, \ldots, j_{D}} \prod_{l} \beta_{2}\left(t / h_{t}-j_{l}\right) .
\end{aligned}
$$

From Lemma B.1, it can be proved that $\prod_{l} \beta_{2}\left(.-j_{l}\right)$ is not identically null if and only if $j_{1}, \ldots, j_{D} \in F_{D}$. As $\beta_{2}$ is a B-spline, it also satisfies the partition of unity property, $\sum_{j} \beta_{2}\left(.-j_{l}\right)=1$, and consequently $\sum_{j_{1}, \ldots, j_{D}} \prod_{l} \beta_{2}\left(.-j_{l}\right)=1$. From these two properties, a lower bound can be deduced:

$$
\begin{aligned}
\min _{j_{1}, \ldots, j_{D} \in F_{D}} J_{i_{1}, \ldots, i_{D}, j_{1}, \ldots, j_{D}} & =\min _{j_{1}, \ldots, j_{D} \in F_{D}} J_{i_{1}, \ldots, i_{D}, j_{1}, \ldots, j_{D}} \sum_{j_{1}, \ldots, j_{D}} \prod_{l} \beta_{2}\left(t / h_{t}-j_{l}\right) \\
& =\min _{j_{1}, \ldots, j_{D} \in F_{D}} J_{i_{1}, \ldots, i_{D}, j_{1}, \ldots, j_{D}} \sum_{j_{1}, \ldots, j_{D} \in F_{D}} \prod_{l} \beta_{2}\left(t / h_{t}-j_{l}\right) \\
& \leq \sum_{j_{1}, \ldots, j_{D} \in F_{D}} J_{i_{1}, \ldots, i_{D}, j_{1}, \ldots, j_{D}} \prod_{l} \beta_{2}\left(t / h_{t}-j_{l}\right) \\
& =\sum_{j_{1}, \ldots, j_{D}} J_{i_{1}, \ldots, i_{D}, j_{1}, \ldots, j_{D}} \prod_{l} \beta_{2}\left(t / h_{t}-j_{l}\right) \\
& =J_{i_{1}, \ldots, i_{D}}(t) .
\end{aligned}
$$

Taking the min over $E_{D}$ of this expression and applying Theorem 3.3, the Jacobian can be bounded below:

$$
\min _{\substack{i_{1}, \ldots, i_{D} \in E_{D} \\ j_{1}, \ldots, j_{D} \in F_{D}}} J_{i_{1}, \ldots, i_{D}, j_{1}, \ldots, j_{D}} \leq \min _{i_{1}, \ldots, i_{D} \in E_{D}} J_{i_{1}, \ldots, i_{D}}(t) \leq J(x, t)
$$

The upper bound is derived similarly.

4. A curse of dimensionality. From Theorem 3.3, a sufficient condition on B-spline coefficients to guarantee that the transformation is a diffeomorphism can be deduced. Unfortunately the complexity of the condition does not scale well with the dimensionality $D$. When $D=2$, the number of constraints per node is $4 n^{2}$, where $n$ is the B-spline degree. In Table 1 , the number of constraints per node is indicated as a function of the dimension and the degree.

As one can see, the number of constraints per node grows very fast with the dimension $D$. From a computational point of view, the condition can be used without restriction for $2 \mathrm{D}$ vector fields and actually leads to fast algorithms. Unfortunately, for full 3D transformations, the number of constraints per node to enforce can be prohibitive. 
Table 1

Number of constraints per node given the dimension and the degree of the vector field.

\begin{tabular}{|c|r|r|r|}
\hline Degree & $1 \mathrm{D}$ & $2 \mathrm{D}$ & $3 \mathrm{D}$ \\
\hline 1 & 1 & 4 & 64 \\
2 & 1 & 16 & 2744 \\
3 & 1 & 36 & 27000 \\
4 & 1 & 64 & 140608 \\
\hline
\end{tabular}

5. Sharpness of the sufficient conditions. The three invertibility conditions from [14], [15], or the present paper are sufficient conditions. This means that when they are satisfied, the vector field is guaranteed to be invertible, but this also means that not all of the B-spline diffeomorphisms satisfy these conditions. If the condition is too restrictive, the search space may not be large enough to contain the diffeomorphism of interest.

Evaluating the size of the search space defined by these conditions is not straightforward. However, one can get an idea of the sharpness of the conditions by considering affine transforms. The analysis using affine transforms is relevant for two reasons. First, affine transformations can be exactly represented using B-splines:

$$
A x+b=\sum_{i}(A(h i)+b) \beta\left(\frac{x}{h}-i\right) .
$$

The B-spline coefficients of $A x+b$ are $c_{i}=A(h i)+b$, and the displacement coefficients are $\tilde{c}_{i}=c_{i}-i h=(A-I)(i h)+b$. When looking at the set of affine transforms that satisfy a condition, one can get an idea of the range of deformations that satisfy the condition. Then, as B-splines have a compact support, their influence is local, so the analysis considering global transforms such as affine ones can be applied locally and in fact gives some clues about the local deformations that satisfy the condition. The locality of the analysis is defined by the node spacing.

Choi-Lee condition. In [14], a bound on each component of the displacement field coefficients is derived that guarantees that the B-spline transform is invertible:

$$
\left\|\tilde{c}_{i}^{l}\right\|_{\infty}<\frac{h_{l}}{K},
$$

where $K \simeq 2.05$ in two dimensions and $K \simeq 2.48$ in three dimensions. If $A=I$, the condition applied to a translation from the identity becomes $|b|<\frac{h_{l}}{K}$. This condition is very restrictive as it allows only small variation from the identity both for the matrix and the translation part of the transform. It can be noted that even transforms as simple as translations can be unable to satisfy it.

Kim-Chun-Fessler condition. In [28] and [15] the invertibility is guaranteed by bounding the finite differences in every direction of the displacement field coefficients:

$$
\left\|\tilde{c}_{i}-\tilde{c}_{i-e_{l}}\right\|_{\infty}<K h_{l},
$$

where $K=\frac{1}{D}$. When applied to the affine transform $A x+b$, the condition becomes

$$
\left\|(A-I) e_{l}\right\|_{\infty}<K \text {. }
$$


In other words, $A$ is in the ball of center $I$ and radius $K$ for the matrix norm $\|M\|=$ $\max _{i j}\left|m_{i j}\right|$. This condition is a clear improvement over the previous one as all translations are now accepted. However, the affine matrix must remain close enough to the identity.

The new condition. In this work, the invertibility is guaranteed by keeping the Jacobian coefficients positive:

$$
J_{i_{1}, \ldots, i_{D}}>0 .
$$

When applied to the affine transform $A x+b$, the condition simply becomes

$$
\operatorname{det}(A)>0
$$

All the invertible affine transforms satisfy the new condition. This confirms that our condition is sharp, as the deformation can be as close to an invertible affine transform as needed. This will be visually confirmed in an example in the numerical experiments in section 7.1.

\section{Application to nonrigid registration and interslice interpolation.}

6.1. Nonrigid registration algorithm. B-spline nonrigid registration is usually solved through the resolution of an optimization problem. In this work, it will be a constrained problem:

$$
\min _{g(c) \leq 0} F_{\lambda}(c)=F(c)+\lambda R(c),
$$

where $F$ is the data fitting term of the cost function, $R$ the regularization term, $g$ the constraints, and $c$ the B-spline coefficients of the transformation. Note that only the coefficients inside the image are estimated, while coefficients outside the reference image are kept fixed; i.e., $c_{i}=A(i h)+b$, where $A$ and $b$ define an affine transform which can be the identity or an affine transform from a potential preliminary affine registration step. Many data terms have been proposed for $F$, from a simple sum of squared differences between the deformed floating image and the reference image [41] to the opposite of their mutual information when registering images of different modality [31], [43]. In this work, a least squares cost function has been used within a multiresolution framework.

The diffeomorphic constraints $g$ are given by Theorem 3.3: the coefficient Jacobians should be above a given threshold $\epsilon_{d}$ :

$$
i_{1}, \ldots, i_{D} \in E_{D}: g_{i_{1}, \ldots, i_{D}}(c)=\epsilon_{d}-J_{i_{1}, \ldots, i_{D}} .
$$

As in [38], the constrained problem is solved by the multipliers method. The augmented Lagrangian is considered:

$$
L_{r}(c, \mu)=F_{\lambda}(c)+\mu \tilde{g}(c)+\frac{r}{2}\|\tilde{g}(c)\|^{2},
$$

where $\mu$ is the vector of the Lagrange multipliers, $r$ is a penalization coefficient, and $\tilde{g}(c)=$ $\max \left(g(c),-\frac{\mu}{r}\right)$. The problem is solved by alternatively minimizing with respect to $x$ and updating $\mu$.

At the end of the algorithm, Jacobian coefficients will be greater than $\epsilon_{d}-C_{\text {stop }}$. By choosing $C_{\text {stop }}<\epsilon_{d}$, the output transformation will be a diffeomorphism. Values of Jacobian coefficients between $C_{\text {stop }}$ and $\epsilon_{d}$ will only be penalized, and Jacobian coefficients are free 


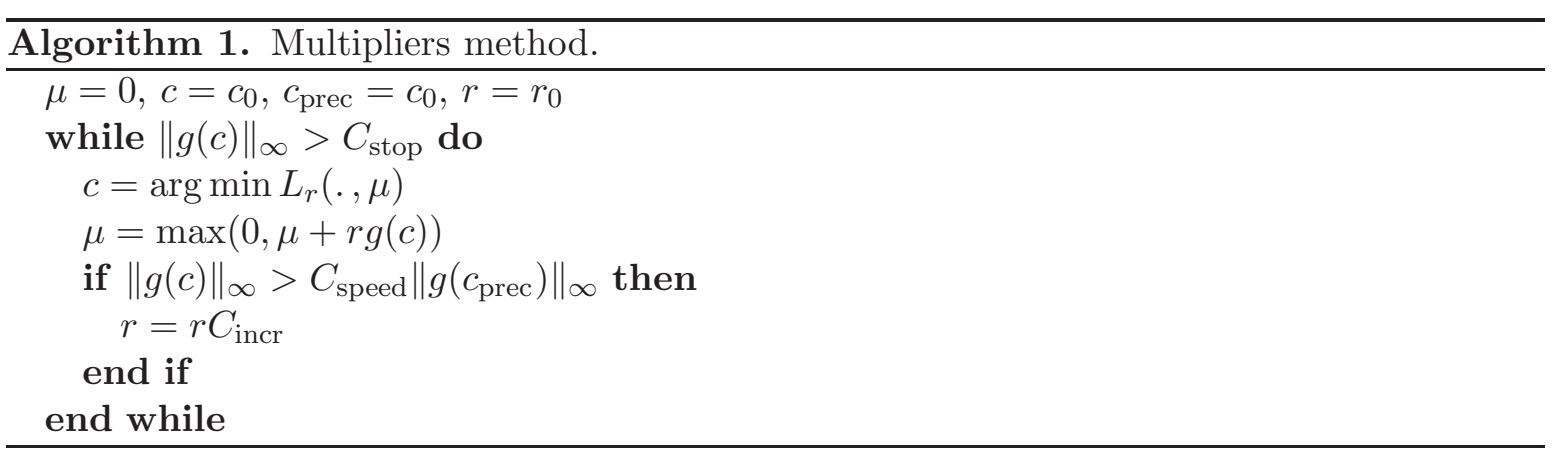

when above $\epsilon_{d}$. In our implementation, $C_{\text {stop }}$ has been set to $\frac{\epsilon_{d}}{2}$. For more insight into the augmented Lagrangian and the multipliers method, please refer to [17], [11], [18], [2], [23].

The unconstrained inner optimization procedure used is the limited memory BFGS (LBFGS) method, which is a quasi-Newton method requiring a low memory size (see [24] or [30]). Derivatives of the cost function can be found in [38] or [29], and derivatives of the constraints are given in Appendix C.

6.2. Interslice interpolation. Diffeomorphic nonrigid registration can be used advantageously in the context of interslice interpolation. Instead of using only the pixel positions to interpolate the intensity of an image between two slices of a 3D image, nonrigid registration can incorporate the image content into the interpolation process as in [35], [6], or [19]. If a transformation $T$ that maps one slice, $I_{0}$, to the next, $I_{1}$, is known, then

$$
I_{1}(x)=I_{0}\left(T^{-1}(x)\right),
$$

and given that

$$
I_{0}(x)=I_{0}\left(I d^{-1}(x)\right),
$$

the interpolation of the images between $I_{0}$ and $I_{1}$ can be done by interpolating the transformation between the identity and $T$. Nonrigid registration offers an approximate solution to the transformation $T$; the problem is to have a solution such that the interpolated transformations are invertible.

The transformation to a slice between these two slices is defined as the linear interpolation between the identity and $T$ :

$$
T_{\alpha}(x)=(1-\alpha) x+\alpha T(x),
$$

where $\alpha$ is a parameter in $[0,1]$ to locate the slice. $T_{\alpha}$ can be seen as a spatio-temporal Bspline vector field, where $\alpha$ is the time variable and the degree in the time dimension is 1 . Note that the identity is a B-spline vector field with displacement coefficients all null, and its Jacobian coefficient matrix is the identity. Let's apply Theorem 3.5: if, for all $i, j \in E_{2}$, the four determinants

$$
\begin{array}{ll}
\operatorname{det}\left(d_{i}^{1}, d_{j}^{2}\right), & \operatorname{det}\left(e_{1}, d_{j}^{2}\right), \\
\operatorname{det}\left(d_{i}^{1}, e_{2}\right), & \operatorname{det}\left(e_{1}, e_{2}\right)
\end{array}
$$

are positive, then $T_{\alpha}$ is invertible for all $\alpha \in[0,1]$. In other words, the determinant as well as the diagonal elements of the Jacobian coefficient matrices should be positive. 
When $T$ is the result of the registration between $I_{0}$ and $I_{1}$ subject to the former conditions, a first interslice intermediate interpolant can be defined as

$$
I_{0}(x, \alpha)=I_{0}\left(T_{\alpha}^{-1}(x)\right) .
$$

To remain unbiased toward the direction of registration, a second intermediate interpolant $I_{1}(., \alpha)$ is found by swapping the two slices and the final interslice interpolant is found by averaging the two intermediate ones:

$$
I(x, \alpha)=\alpha I_{0}(x, \alpha)+(1-\alpha) I_{1}(x, 1-\alpha) .
$$

\section{Numerical experiments.}

7.1. 2D registration of synthetic images. A comparison of the new diffeomorphic registration with other registration methods from the literature has been done on the classical problem of the registration of a disk to a $C$ shape. The images are binary, and the size of both is $300 \times 300$ pixels. The coefficient Jacobian constraint (CJ) presented in this work is compared to unconstrained registration (NO), to a registration with the bound constraint (BC) of [14], to the bound on finite differences constraint (DC) of [28], [15], and to the constraint on pixelwise sampled Jacobian (SJ) of [38]. To these B-spline based registration methods we added the diffeomorphic demons (DD) of [49]. Node spacing for B-spline registrations was $6 \times 6$, and third degree B-splines were used for interpolate the image and to model the transformation. For DD, the fluid-like and diffusion-like $\sigma$ were both set to 1 , and the maximum step length was 2 . The registration was done using four scales. The maximum number of iterations was set on each scale from coarse to fine to $15,10,5$, and 5 for DD and 50, 25, 10, and 15 for the other methods. The multipliers method was run until convergence, i.e., until constraints satisfaction.

The different methods are visually compared by mapping the input floating image and the disk, as well as a grid using the transformation found by each method. The contours of the reference image are overlaid on each image, and the results are presented in Figure 1. Simple statistics on the Jacobian map of each method (range and percentage of negative Jacobians), as well as the CPU time of the registration, are presented in Table 2.

Except for BC and DC, all methods are able to produce a visually correct registration: the disk is perfectly transformed into the $\mathrm{C}$ shape. By imposing a bound on the displacement coefficient or its finite differences, $\mathrm{BC}$ and $\mathrm{DC}$ guarantee that the resulting transformation is a diffeomorphism. The cost of this guarantee is a reduction of the search space so important that large deformations cannot be recovered. Note that the results obtained in this work are similar to those of [15]. In the latter, the authors also try to get a perfect match by composing the transformation. Thirty-five compositions of $\mathrm{BC}$ or three compositions of DC were required to correctly register the disk to the C. Using the new constraints CJ, the diffeomorphic property is guaranteed, but large deformations are recovered directly, without the need to compose several vector fields. This allows us to keep the B-spline representation as the output of the final transformation.

The unconstrained registration indeed does a good job of matching the two images. However as one can see on the deformation of the grid, serious foldings are present in the transformation. One can also see that Jacobians are negative on $15 \%$ of the pixels. 


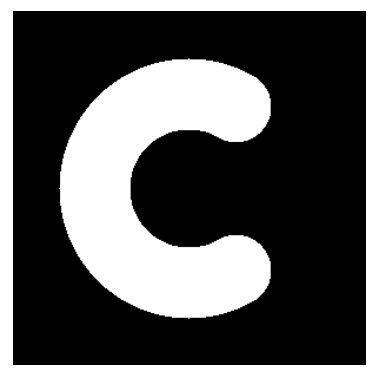

(a) Reference image

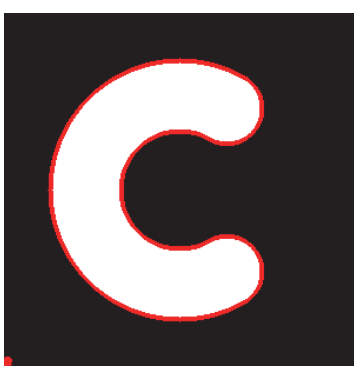

(c) No constraint (NO)

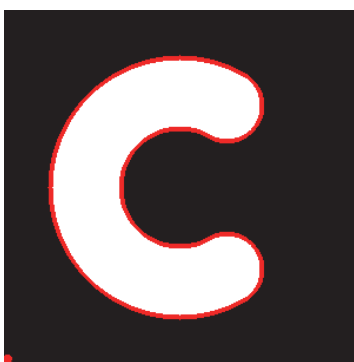

(g) Sampled Jacobian (SJ)

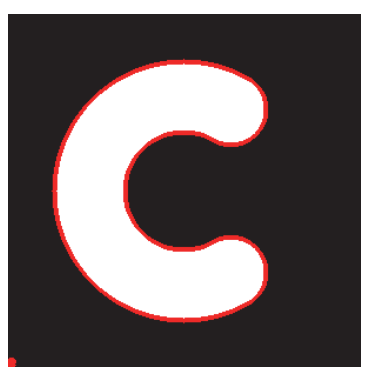

$(\mathrm{k})$ demons (DD)

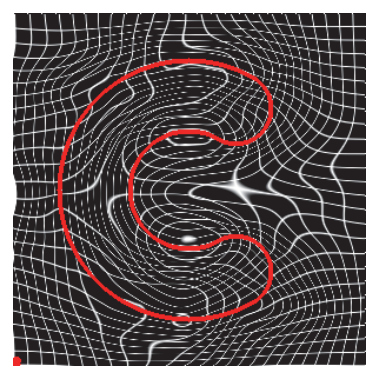

(d) No constraint (NO)

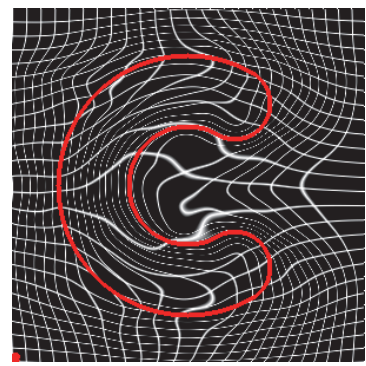

(h) Sampled Jacobian (SJ)

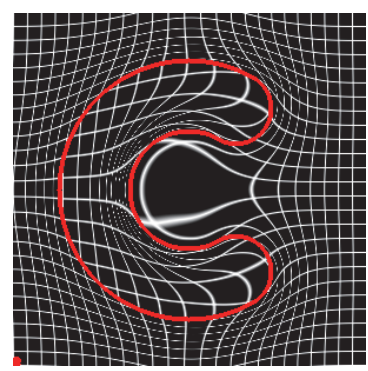

(l) Diffeomorphic demons (DD)

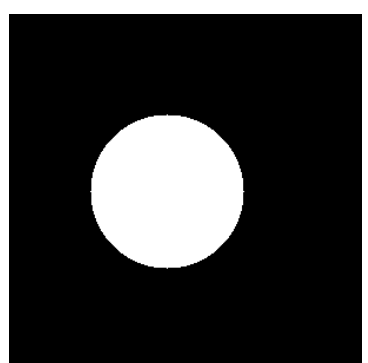

(b) Floating image

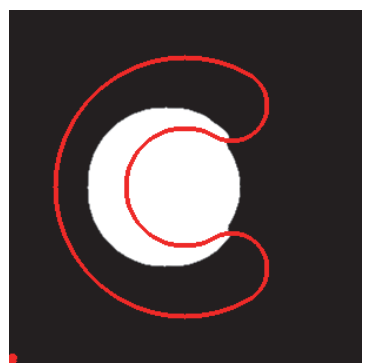

(e) Choi-Lee (BC)

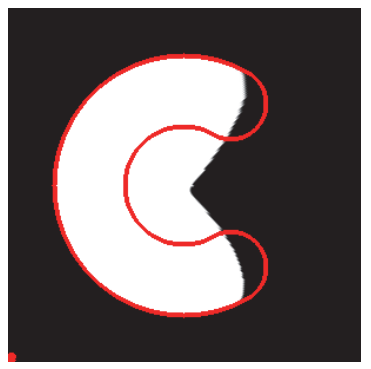

(i) Kim-Chun-Fessler (DC)

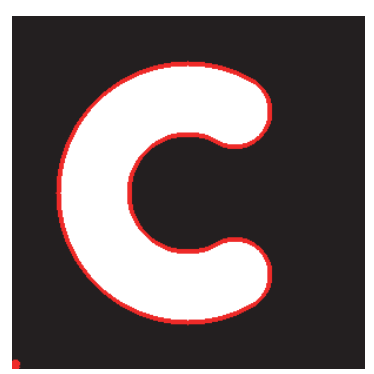

(m) New constraint (CJ)

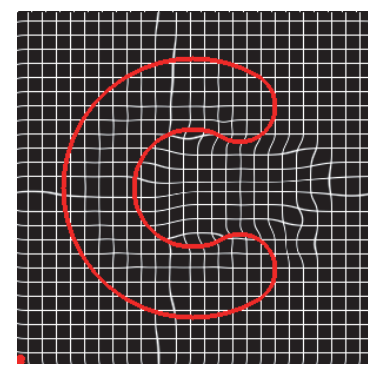

(f) Choi-Lee (BC)

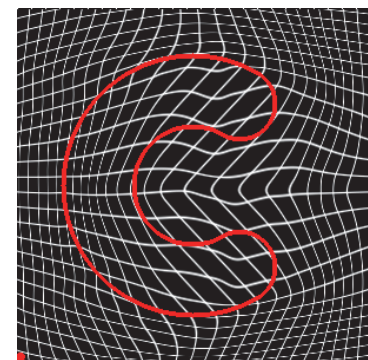

(j) Kim-Chun-Fessler (DC)

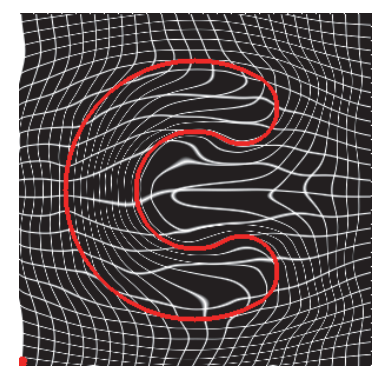

(n) New constraint (CJ)

Figure 1. Results of the registration for different methods: the floating image and a grid are mapped through the transformation. A contour image of the reference is overlaid on these images.

No folding is apparent on the deformation grid image for the SJ method, while at the same time the two images are correctly matched. It can be seen from Table 2 that Jacobians are 
Table 2

Extrema of the Jacobians, percentage of negative Jacobians, and CPU time (in seconds) for the different methods.

\begin{tabular}{|l|r|r|r|r|}
\hline Method & $\min J$ & $\max J$ & $J \leq 0$ & Time \\
\hline Unconstrained (NO) & -2.703 & 5.69 & 14.5 & 4 \\
Sampled Jacobian (SJ) & 0.008 & 3.10 & 0 & 14 \\
Choi-Lee (BC) & 0.220 & 1.49 & 0 & 6 \\
Kim-Chun-Fessler (DC) & 0.128 & 3.78 & 0 & 11 \\
New constraint (CJ) & 0.011 & 3.46 & 0 & 13 \\
Diffeomorphic demon (DD) & -1.871 & 8.06 & 5.7 & 12 \\
\hline
\end{tabular}

positive on all pixels (as imposed by the constraints). If this point is of important practical value (for example, for further statistical analysis of Jacobian maps), this does not guarantee that the transformation found by this method is a diffeomorphism as the Jacobian map can take negative values between pixels.

DD also correctly registers the two images while apparently avoiding foldings in the transformation. However, as seen in Table 2, $6 \%$ of the Jacobians are negative in the image with values down to -1.87 . As opposed to B-spline based registration, analytical expression of the Jacobians is not available for DD, and Jacobians have to be computed using a finite different scheme. As pointed out in [49], the numerical computation of Jacobians is not consistent with the theory behind DD. This is why negative Jacobians can be obtained even if the transformation is diffeomorphic. Note also that if the maximum length of an update vector parameter of DD is too large, the transformation might not be invertible at all.

Regarding the computation time, the registration is performed in about $5 \mathrm{~s}$ for $\mathrm{BC}$ and $\mathrm{NO}$ and in around $12 \mathrm{~s}$ for the other methods. This makes the new diffeomorphic registration in two dimensions competitive with DD, which is considered a fast registration method. Note that DD was run using the $\mathrm{C}++$ software provided with the Insight Toolkit (ITK) library and the other algorithms are based on a MATLAB/C implementation ${ }^{1}$ and can be further optimized. For the registrations using the multipliers method to solve the constrained problem (SJ, DC, and CJ), convergence was reached after four to six outer iterations. Note also that the number of inner iterations for all methods is manually set and a robust stopping criterion would be of great interest in reducing the computational time.

7.2. Topology preservation experiment. In this experiment, the registration between two synthetic images is performed for unconstrained registration and the new diffeomorphic registration. The diffeomorphic registration was performed using several values for the Bspline degree and for the Jacobian lower bound (the $\epsilon_{d}$ parameter of (6.1)). To observe the topology preservation property of diffeomorphic registration and its behavior in the presence of nondiffeomorphic structures, the reference image (two balls) and the floating image (an ellipse) have been chosen such that they cannot be matched using a diffeomorphic transformation.

Node spacing for B-spline registrations was $6 \times 6$. The registration was done using four scales. The maximum number of inner iterations was set on each scale from coarse to fine to $30,30,20$, and 8 . The multipliers method was run until convergence.

The results are visually compared by mapping the input floating image, the ellipse, as well

\footnotetext{
${ }^{1}$ The code is based on the library provided at http://web.eecs.umich.edu/ fessler/code/index.html.
} 


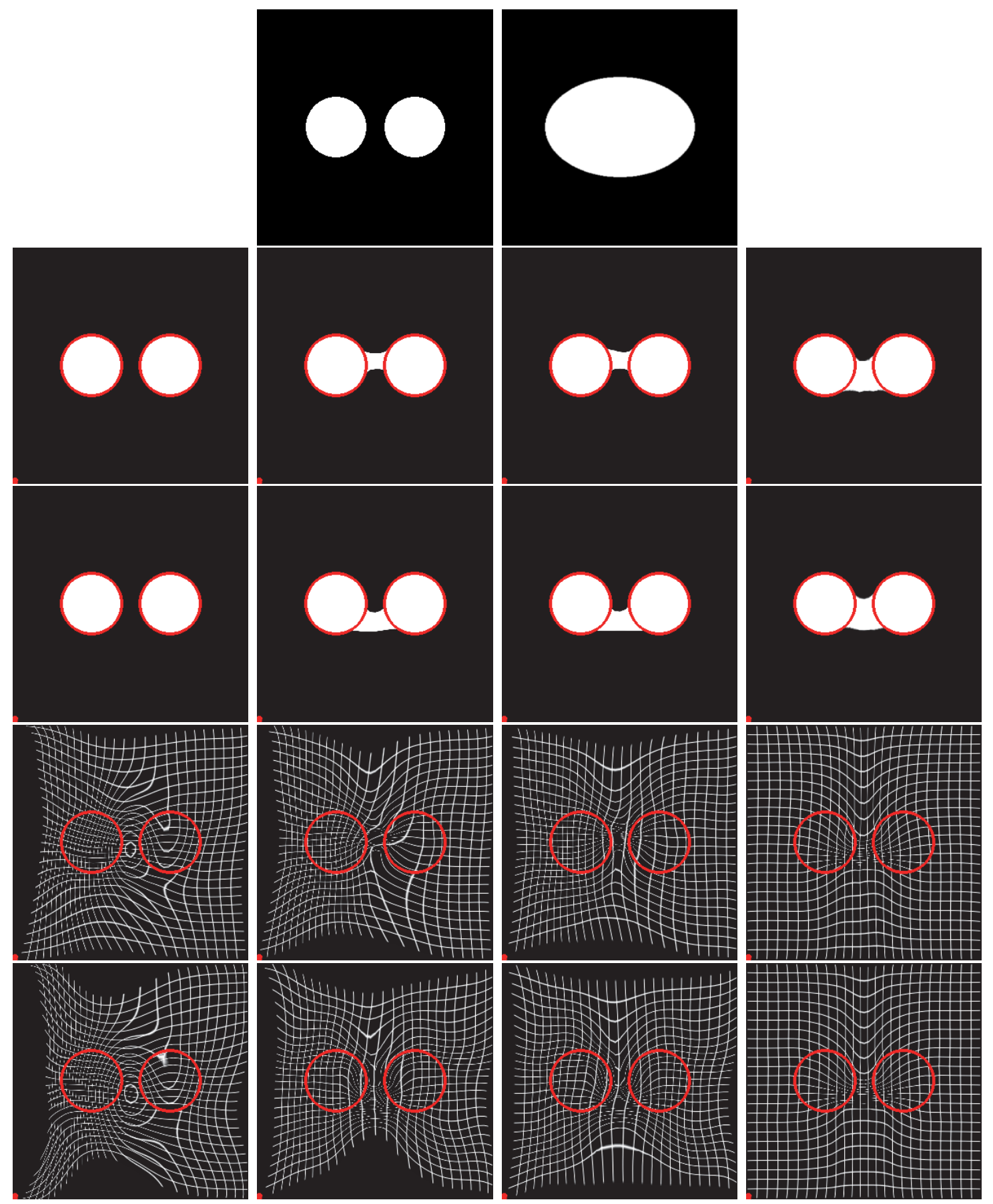

Figure 2. Results of the registration for unconstrained registration and constrained registration for several values of the B-spline degree and the determinant lower bound $\epsilon_{d}$. The reference and floating images are in the top row; in rows 2-3 are the deformed image for B-spline degrees 1 and 3 and in rows 4-5 the deformed grid for degrees 1 and 3 . The first column shows the results of unconstrained registration, followed by the results of the new constrained registration with the $\epsilon_{d}$ parameter sets to $0.001,0.25$, and 1. 
Table 3

Number of multipliers method (MM) iterations, CPU time, and minimum Jacobians for different spline degree and $\epsilon_{d}$ values.

\begin{tabular}{||l||r|r|r||r|r|r||r|r|r||}
\hline \multicolumn{1}{||c||}{} & \multicolumn{3}{|c|}{ MM iterations } & \multicolumn{3}{c|}{ CPU time } & \multicolumn{3}{|c||}{ min J } \\
\hline$\epsilon_{d}$ & deg. 1 & deg. 2 & deg. 3 & deg. 1 & deg. 2 & deg. 3 & deg. 1 & deg. 2 & deg. 3 \\
\hline NO & & & & 1.91 & 1.94 & 1.96 & -3.7342 & -7.7438 & -3.0352 \\
0.0001 & 7 & 20 & 16 & 6.40 & 13.74 & 14.01 & 0.0013 & 0.0001 & 0.0003 \\
0.001 & 9 & 36 & 18 & 10.06 & 23.11 & 14.85 & 0.0022 & 0.0010 & 0.0011 \\
0.01 & 6 & 23 & 8 & 5.96 & 15.52 & 8.08 & 0.0136 & 0.0074 & 0.0107 \\
0.10 & 7 & 8 & 5 & 6.26 & 6.96 & 6.09 & 0.0492 & 0.0359 & 0.0405 \\
0.25 & 4 & 7 & 5 & 4.39 & 6.50 & 6.05 & 0.0781 & 0.0868 & 0.0816 \\
0.50 & 1 & 2 & 4 & 3.04 & 3.86 & 5.26 & 0.1687 & 0.2147 & 0.2338 \\
0.75 & 3 & 1 & 1 & 4.09 & 3.33 & 3.48 & 0.4682 & 0.4432 & 0.4219 \\
1 & 1 & 1 & 1 & 2.88 & 3.23 & 3.42 & 0.7180 & 0.6961 & 0.6199 \\
& 1 & 1 & 1 & 2.82 & 2.88 & 3.01 & 0.8255 & 0.7412 & 0.7276 \\
\hline
\end{tabular}

as a grid using the transformations found. The contours of the reference image are overlaid on each image, and the results are presented in Figure 2. The number of outer iterations of the multipliers method, the CPU time, and the minimum Jacobian are presented in Table 3.

One can see in Figure 2 that the unconstrained registration gives a perfect match of the two images, but consequently the topology of the floating image is changed by the deformation. Using the constrained registration, the matching is good everywhere but on a ribbon between the two balls. This ribbon makes the topology of the floating image equivalent before and after deformation. Its shape seems to be variable, which makes us suspect that the cost function is quite flat in this region and/or has local minima. With the increase on the $\epsilon_{d}$ parameter, one can also notice a small enlargement of the ribbon and that the grid becomes more regular. The change of B-spline degree does not seem to affect the registration very much.

From Table 3, one can see that computational time and the number of outer iterations decrease when $\epsilon_{d}$ is increased. This is due to the value of $C_{\text {stop }}$ (see Algorithm 1), which is set to $\frac{\epsilon_{d}}{2}$. When $\epsilon_{d}$ is increased, the search space is reduced, but the constraints need to be satisfied less strictly to make the transformation diffeomorphic.

Regarding the B-spline degree, the CPU time and number of outer iterations increase somewhat when changing the degree from 1 to 3 . This was expected as when the degree increases, the number of constraints as well as the complexity of the data fitting term also increases. Surprisingly, when using quadratic B-splines, the number of iterations and the CPU time are higher than when cubic B-splines are used. As expected, the Jacobian minimum is correlated to $\epsilon_{d}$. The B-spline degree does not seem to have an influence on the Jacobian minimum.

7.3. Interslice interpolation. The registration based interpolation (RBI) has been compared to a standard linear interpolation (LI) on in vivo $T_{2}$ magnetic resonance images of the brain. The data were downloaded from the NAMIC website. ${ }^{2}$ The image is $256 \times 256 \times 176$ voxels and voxel size is $1 \mathrm{~mm} \times 1 \mathrm{~mm} \times 1 \mathrm{~mm}$. In real life clinical protocol, it is not unusual to have a $3 \mathrm{~mm}$ or even $5 \mathrm{~mm}$ interslice spacing for a $T_{2}$ image. Two slices $5 \mathrm{~mm}$ apart have been

\footnotetext{
${ }^{2}$ See http://hdl.handle.net/1926/1687.
} 
Table 4

$M A D$ for each slice between $L I$ and $R B I$.

\begin{tabular}{|c|r|r|}
\hline Slice & \multicolumn{1}{|c|}{ LI } & \multicolumn{1}{|c|}{ RBI } \\
\hline 0 & 0.00 & 0.00 \\
1 & 18.08 & 15.55 \\
2 & 27.01 & 20.93 \\
3 & 27.65 & 20.95 \\
4 & 19.06 & 15.38 \\
5 & 0.00 & 0.00 \\
\hline
\end{tabular}
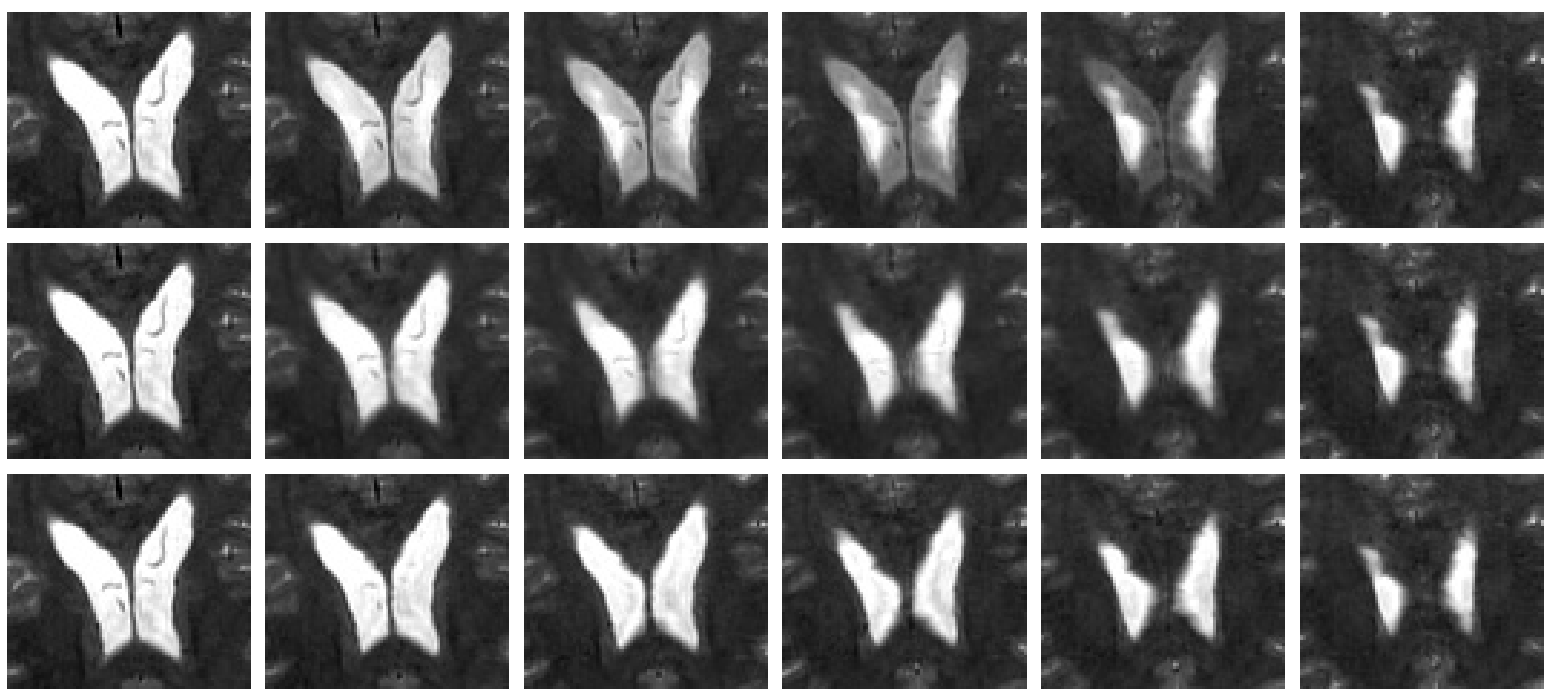

Figure 3. Interslice interpolation (zoom around the ventricles). The first and last columns are the input slices of the interpolation, and the middle columns are the interpolated slices. The LI results are in the first row, the RBI results are in the second row and the third row is the ground truth.

extracted and used to test the interslice interpolation.

The mean of absolute differences (MAD) between the interpolated images $\left(I_{i}\right)$ and the ground truth $\left(I_{g}\right)$ is used to compare the LI and RBI:

$$
M A D=\frac{1}{N} \sum_{x}\left|I_{i}(x)-I_{g}(x)\right|
$$

The MAD for each slice is given in Table 4.

As expected, for both methods, the error is null on the first and last slices and increases with the distance from the input slices. One can notice that a better result is obtained with RBI for all slices and that the best improvement is obtained for middle slices (slices 2 and 3 ).

Figure 3 presents the result of the two interpolations in the ventricular region. In this region, the deformation of the ventricles is important between the two slices, and the two images are very different. This is why LI does a poor job. It can be clearly seen that the resulting intensity around the ventricles is a mix of the two input images. With RBI, as the input images are deformed before they are mixed, such an artifact does not occur, and the interpolated images keep sharp boundaries. 
8. Conclusion. A new sufficient condition to guarantee the invertibility of a B-spline vector field $T$ is presented in this work. The condition is also extended to a spatio-temporal vector field. The condition, that Jacobians of the B-spline coefficient should be positive, can be seen as the natural extension of the continuous condition for all $x, \operatorname{det}\left(T^{\prime}(x)\right)>0$ to the B-spline coefficients. The infinite number of inequality constraints in the continuous space is reduced to a finite set of inequality constraints.

A diffeomorphic registration method has also been proposed using the sufficient condition as inequality constraint in a constrained optimization modelization of the registration process. This diffeomorphic registration has further been used to propose a high quality interslice interpolation.

The main limitation of our proposal can be resumed as a curse of dimensionality: the number of constraints increases very quickly with the dimension of the vector field. In practical application, we would say that this limits the usability of our proposal to a $2 \mathrm{D}$ vector field, $2 \mathrm{D}+t$ spatio-temporal vector field, or inplane constrained $3 \mathrm{D}$ deformation. For full $3 \mathrm{D}$ transformation, the the number of constraints per node necessary to guarantee invertibility makes it unusable. Despite this restriction, our method can still be applied to a large number of problems. For example, it can be used for atlas based segmentation of 2D images [40]; to segment 3D images with 2D atlases as in [21]; to correct the geometric distortion occurring in the phase direction of echo planar images [3], [1]; to perform high quality slice interpolation [35], [6], [19]; for cortical surface matching [27], [26]; or for transmission electron microscopy reconstruction [20].

Numerical experiments have been performed on synthetic and real life problems. The $2 \mathrm{D}$ diffeomorphic registration has been compared to different state-of-the-art methods on the standard registration problem between a disk and a $\mathrm{C}$ shape. Our method is the only one with all of the following properties: the registration is visually correct, the transformation is truly diffeomorphic, the Jacobian map can be computed exactly, and only positive Jacobians are obtained. Regarding the computation time, for a 2D problem, our method is competitive with DD, which is considered a fast method.

Slice interpolation experiments have also been performed. For this more realistic experiment, our diffeomorphic registration has been used to interpolate the data between two slices of a $T_{2}$ weighted image of the brain. Interpolation errors have been measured, and a comparison has been made to a linear interpolation of the slices. Numerical results as well as visual inspection clearly show that our method offers a strong improvement over LI.

Appendix A. Main B-spline properties. In this section, the definition and main properties of B-splines are presented. For more insight on B-splines, please refer, for example, to [46].

Definition A.1. The $1 D$ 0th-degree B-spline $\beta_{0}$ is the characteristic function of the interval $\left[-\frac{1}{2}, \frac{1}{2}\left[\right.\right.$. The nth-degree B-spline $\beta_{n}$ is obtained by convolving this function with itself $n$ times:

$$
\beta_{n}=\beta_{n-1} * \beta_{0} .
$$

Here we summarize some basic properties of the 1D B-splines used in this paper:

1. $\beta_{n}$ is an $n$ th-degree piecewise polynomial function.

2. $\beta_{n}(t) \geq 0$.

3. The support of $\beta_{n}$ is $\left[-\frac{n+1}{2}, \frac{n+1}{2}\right]$. 
4. For all $t, \sum_{i} \beta_{n}(t-i)=1$.

5. $\beta_{n}^{\prime}(t)=\beta_{n-1}\left(t+\frac{1}{2}\right)-\beta_{n-1}\left(t-\frac{1}{2}\right)$.

6. If $f(t)=\sum_{i} c_{i} \beta_{n}(t-i)$, then for all $t, \min _{i} c_{i} \leq f(t) \leq \max _{i} c_{i}$.

Appendix B. B-spline overlap lemmas. Let $\beta$ be the $n$-degree $1 \mathrm{D}$ B-spline and $\beta^{-}$the spline of degree $n-1$. $\beta$ is null everywhere except on the interval $\left.\frac{n+1}{2} \times\right]-1,1$. Consequently, for $(a, b) \in \mathbb{Z}^{2}$ and $h>0, \beta(t / h-a)$ does not vanish only on the interval

$$
h \times\left(a+\frac{n+1}{2} \times\right]-1,1[),
$$

and $\beta^{-}\left(t / h-a+\frac{1}{2}\right)$ is null everywhere except on

$$
h \times\left(a-\frac{1}{2}+\frac{n}{2} \times\right]-1,1[) .
$$

Lemma B.1. There exists $t$ such that $\beta(t / h-a) \beta(t / h-b) \neq 0$ if and only if $b-a \in[-n, n]$. Proof. Take such a $t$; then there exists $(\lambda, \mu) \in]-1,1\left[{ }^{2}\right.$ such that

$$
t=h \times\left(a+\lambda \frac{n+1}{2}\right)=h \times\left(b+\mu \frac{n+1}{2}\right) .
$$

We deduce that

$$
b-a=(\lambda-\mu) \frac{n+1}{2} .
$$

As $(\lambda-\mu) \in]-2,2[$, we can conclude that

$$
b-a \in[-n, n]
$$

Lemma B.2. There exists $t$ such that $\beta^{-}\left(t / h-a+\frac{1}{2}\right) \beta(t / h-b) \neq 0$ if and only if $b-a \in$ $[-n, n-1]$.

Proof. If the B-spline product above is not null for a given $t$, this $t$ can be expressed as

$$
t=h \times\left(b+\mu \frac{n+1}{2}\right)=h \times\left(a-\frac{1}{2}+\lambda \frac{n}{2}\right)
$$

with $(\lambda, \mu) \in]-1,1\left[^{2}\right.$. We deduce that

$$
\left.b-a=\lambda \frac{n}{2}-\frac{1}{2}-\mu \frac{n+1}{2} \in\right]-n-1, n[
$$

and finally

$$
b-a \in[-n, n-1] .
$$

Appendix C. Constraint derivatives. In this appendix, the derivatives of the constraints in two dimensions with respect to a B-spline coefficient $c_{k, q}$, where $k$ is the B-spline index and 
$q$ is the component index, are given. Let us start with the derivatives of the finite difference coefficient,

$$
\begin{aligned}
\frac{\partial d_{i}^{l}}{\partial c_{k, q}} & =\frac{\partial}{\partial c_{k, q}}\left(\frac{c_{i}-c_{i-e_{l}}}{h_{l}}\right) \\
& =\frac{\delta_{i k}-\delta_{i-e_{l}, k}}{h_{l}} e_{q}
\end{aligned}
$$

where $\delta$ is the Kronecker symbol and $e_{q}$ is the $q$ th vector of the canonical basis.

In two dimensions, the Jacobian coefficient derivatives are

$$
\begin{aligned}
\frac{\partial J_{i j}}{\partial c_{k}^{q}} & =\frac{\partial}{\partial c_{k, q}}\left|d_{i}^{1}, d_{j}^{2}\right| \\
& =\left|\frac{\partial d_{i}^{1}}{\partial c_{k, q}}, d_{j}^{2}\right|+\left|d_{i}^{1}, \frac{\partial d_{i}^{2}}{\partial c_{k, q}}\right| \\
& =\frac{\delta_{i k}-\delta_{i-e_{l}, k}}{h_{1}}\left|e_{q}, d_{j}^{2}\right|+\frac{\delta_{j k}-\delta_{j-e_{l}, k}}{h_{2}}\left|d_{i}^{1}, e_{q}\right| \\
& =(-1)^{q+1}\left(\frac{\delta_{i k}-\delta_{i-e_{l}, k}}{h_{1}} d_{j, \bar{q}}^{2}+\frac{\delta_{j k}-\delta_{j-e_{l}, k}}{h_{2}} d_{i, \bar{q}}^{1}\right),
\end{aligned}
$$

where $\bar{q}=3-q$. One can see that each Jacobian coefficient has an influence on only four gradient components.

\section{REFERENCES}

[1] J. L. R. Andersson, C. Hutton, J. Ashburner, R. Turner, And K. Friston, Modeling geometric deformations in EPI time series, NeuroImage, 13 (2001), pp. 903-919.

[2] R. Andreani, E. G. Birgin, J. M. Martínez, and M. L. Schuverdt, On augmented Lagrangian methods with general lower-level constraints, SIAM J. Optim., 18 (2007), pp. 1286-1309.

[3] S. Ardekani and U. Sinha, Geometric distortion correction of high-resolution 3 T diffusion tensor brain images, Magn. Reson. Med., 54 (2005), pp. 1163-1171.

[4] J. Ashburner, A fast diffeomorphic image registration algorithm, NeuroImage, 38 (2007), pp. 95-113.

[5] J. Ashburner, C. Hutton, R. Frackowiak, I. Johnsrude, C. Price, and K. Friston, Identifying global anatomical differences: Deformation-based morphometry, Hum. Brain Mapp., 6 (1998), pp. 348 357.

[6] H. Atoui, S. Miguet, And D. Sarrut, A fast morphing-based interpolation for medical images: Application to conformal radiotherapy, Image Anal. Stereol., 25 (2006), pp. 95-103.

[7] G. Auzias, J. Lefìvre, A. Le Troter, C. Fischer, M. Perrot, J. Regis, and O. Coulon, Modeldriven harmonic parameterization of the cortical surface: HIP-HOP, IEEE Trans. Med. Imag., 32 (2013), pp. 873-887.

[8] B. B. Avants, C. L. Epstein, M. Grossman, and J. C. Gee, Symmetric diffeomorphic image registration with cross-correlation: Evaluating automated labeling of elderly and neurodegenerative brain, Med. Image Anal., 12 (2008), pp. 26-41.

[9] M. F. Beg, M. I. Miller, A. Trouvé, And L. Younes, Computing large deformation metric mappings via geodesic flows of diffeomorphisms, Int. J. Comput. Vision, 61 (2005), pp. 139-157.

[10] S. Belongie, J. Malik, And J. Puzicha, Shape matching and object recognition using shape contexts, IEEE Trans. Pattern Anal. Mach. Intell., 24 (2002), pp. 509-522.

[11] E. G. Birgin, R. A. Castillo, and J. M. Martĩnez, Numerical comparison of augmented Lagrangian algorithms for nonconvex problems, Comput. Optim. Appl., 31 (2005), pp. 31-55. 
[12] C. Broit, Optimal Registration of Deformed Images, Ph.D. thesis, University of Pennsylvania, Philadelphia, PA, 1981.

[13] Y. CaO, M. I. Miller, R. L. Winslow, And L. Younes, Large deformation diffeomorphic metric mapping of fiber orientations, in Proceedings of the Tenth IEEE International Conference on Computer Vision, IEEE Computer Society, Washington, DC, 2005, pp. 1379-1386.

[14] Y. Choi And S. LeE, Injectivity conditions of $2 D$ and $3 D$ uniform cubic B-spline functions, Graph. Models, 62 (2000), pp. 411-427.

[15] S. Y. Chun AND J. A. Fessler, A simple regularizer for B-spline nonrigid image registration that encourages local invertibility, IEEE J. Sel. Topics Signal Process., 3 (2009), pp. 159-169.

[16] M. K. Chung, K. J. Worsley, T. Paus, C. Cherif, D. L. Collins, J. N. Giedd, J. L. Rapoport, And A. C. Evans, A unified statistical approach to deformation-based morphometry, NeuroImage, 14 (2001), pp. 595-606.

[17] A. R. Conn, N. Gould, A. Sartenaer, And Ph. L. Toint, Convergence properties of an augmented Lagrangian algorithm for optimization with a combination of general equality and linear constraints, SIAM J. Optim., 6 (1996), pp. 674-703.

[18] A. R. Conn, N. Gould, And Ph. L. Toint, A globally convergent Lagrangian barrier algorithm for optimization with general inequality constraints and simple bounds, Math. Comput., 66 (1997), pp. 261288.

[19] L. Cordero-Grande, G. Vegas-Sanchez-Ferrero, P. Casaseca-de-la Higuera, and C. AlBerola-Lopez, A Markov random field approach for topology-preserving registration: Application to object-based tomographic image interpolation, IEEE Trans. Image Process., 21 (2011), pp. $2047-$ 2061.

[20] J. Dauguet, D. Bock, R. C. Reid, And S. K. Warfield, Alignment of large image series using cubic B-splines tessellation: Application to transmission electron microscopy data, in Medical Image Computing and Computer-Assisted Intervention-MICCAI 2007, Springer-Verlag, Berlin, Heidelberg, 2007, pp. 710-717.

[21] F. Ding, W. K. Leow, And S.-C. Wang, Segmentation of 3D CT volume images using a single 2D atlas, in Computer Vision for Biomedical Image Applications, Springer-Verlag, Berlin, Heidelberg, 2005, pp. 459-468.

[22] B. Fischl, M. I. Sereno, R. B. H. Tootell, And A. M. Dale, High-resolution intersubject averaging and a coordinate system for the cortical surface, Hum. Brain Mapp., 8 (1999), pp. 272-284.

[23] J. Ch. Gilbert, Optimisation différentiable: Théorie et algorithmes, course syllabus, ENSTA, Paris, 2003.

[24] J. C. Gilbert and C. Lemaréchal, Some numerical experiments with variable-storage quasi-Newton algorithms, Math. Programming, 45 (1989), pp. 407-435.

[25] M. Hernandez, M. N. Bossa, And S. Olmos, Registration of anatomical images using geodesic paths of diffeomorphisms parameterized with stationary vector fields, in Proceedings of the IEEE 11th International Conference on Computer Vision, 2007, pp. 1-8.

[26] A. A. Joshi, D. Pantazis, Q. Li, H. Damasio, D. W. Shattuck, A. W. Toga, and R. M. Leahy, Sulcal set optimization for cortical surface registration, NeuroImage, 50 (2010), pp. 950-959.

[27] A. A. Joshi, D. W. Shattuck, P. M. Thompson, And R. M. Leahy, Surface-constrained volumetric brain registration using harmonic mappings, IEEE Trans. Med. Imag., 26 (2007), pp. 1657-1669.

[28] J. KIm, Intensity Based Image Registration Using Robust Similarity Measure and Constrained Optimization: Applications for Radiation Therapy, Ph.D. thesis, University of Michigan, Ann Arbor, MI, 2004.

[29] J. Kybic And M. Unser, Fast parametric elastic image registration, IEEE Trans. Image Process., 12 (2003), pp. 1427-1442.

[30] D. C. Liu AND J. Nocedal, On the limited memory BFGS method for large scale optimization, Math. Programming, 45 (1989), pp. 503-528.

[31] F. Maes, A. Collignon, D. Vandermeulen, G. Marchal, and P. Suetens, Multimodality image registration by maximization of mutual information, IEEE Trans. Med. Imag., 16 (1997), pp. 187-198.

[32] M. I. Miller, G. E. Christensen, Y. Amit, and U. Grenander, Mathematical textbook of deformable neuroanatomies, Proc. Nat. Acad. Sci. USA, 90 (1993), 11944.

[33] O. Musse, F. Heitz, And J.-P. ARmspach, Topology preserving deformable image matching using constrained hierarchical parametric models, IEEE Trans. Image Process., 10 (2001), pp. 1081-1093. 
[34] V. Noblet, C. Heinrich, F. Heitz, And J.-P. Armspach, 3-D deformable image registration: A topology preservation scheme based on hierarchical deformation models and interval analysis optimization, IEEE Trans. Image Process., 14 (2005), pp. 553-566.

[35] G. P. Penney, J. A. Schnabel, D. Rueckert, M. A. Viergever, and W. J. Niessen, Registrationbased interpolation, IEEE Trans. Med. Imag., 23 (2004), pp. 922-926.

[36] D. Rueckert, P. Aljabar, R. A. Heckemann, J. V. Hajnal, and A. Hammers, Diffeomorphic registration using B-splines, in Medical Image Computing and Computer-Assisted InterventionMICCAI 2006, Springer-Verlag, Berlin, Heidelberg, 2006, pp. 702-709.

[37] J. Schaerer, C. Casta, J. Pousin, and P. Clarysse, A dynamic elastic model for segmentation and tracking of the heart in MR image sequences, Med. Image Anal., 14 (2010), pp. 738-749.

[38] M. SDIKA, A fast nonrigid image registration with constraints on the Jacobian using large scale constrained optimization, IEEE Trans. Med. Imag., 27 (2008), pp. 271-281.

[39] M. SDIKA, Combining atlas based segmentation and intensity classification with nearest neighbor transform and accuracy weighted vote, Med. Image Anal., 14 (2010), pp. 219-226.

[40] A. Sotiras, N. Komodakis, G. Langs, and N. Paragios, Atlas-based deformable mutual population segmentation, in Proceedings of the IEEE International Symposium on Biomedical Imaging: From Nano to Macro, 2009, pp. 5-8.

[41] R. Szeliski and J. Coughlan, Spline-Based Image Registration, Technical report 94/1, Digital Equipment Corporation, Cambridge Research Laboratory, Cambridge, MA, 1994.

[42] R. Szeliski And H. Y. Shum, Motion estimation with quadtree splines, IEEE Trans. Pattern Anal. Mach. Intell., 18 (1996), pp. 1199-1210.

[43] P. ThÉvenaz And M. Unser, A pyramid approach to sub-pixel image fusion based on mutual information, in Proceedings of the IEEE International Conference on Image Processing, 1996, pp. 265-268.

[44] J. P. Thirion, Image matching as a diffusion process: An analogy with Maxwell's demons, Med. Image Anal., 2 (1998), pp. 243-260.

[45] A. Trouvé, Diffeomorphisms groups and pattern matching in image analysis, Int. J. Comput. Vision, 28 (1998), pp. 213-221.

[46] M. Unser, Splines: A perfect fit for signal and image processing, IEEE Signal Processing Mag., 16 (1999), pp. 22-38.

[47] M. Unser, A. Aldroubi, And M. Eden, B-spline signal processing. I. Theory, IEEE Trans. Signal Process., 41 (1993), pp. 821-833.

[48] J. Vandemeulebroucke, S. Rit, J. Kybic, P. Clarysse, and D. Sarrut, Spatiotemporal motion estimation for respiratory-correlated imaging of the lungs, Med. Phys., 38 (2011), pp. 166-178.

[49] T. Vercauteren, X. Pennec, A. Perchant, and N. Ayache, Diffeomorphic demons: Efficient nonparametric image registration, NeuroImage, 45 (2009), pp. S61-S72.

[50] T. WAKAHARA AND S. UCHIDA, Hierarchical decomposition of handwriting deformation vector field using $2 D$ warping and global/local affine transformation, in Proceedings of the 2009 10th International Conference on Document Analysis and Recognition (ICDAR'09), IEEE Computer Society, Washington, DC, 2009, pp. 1141-1145.

[51] S. Warfield, A. Robatino, J. Dengler, F. A. Jolesz, and R. Kikinis, Nonlinear registration and template driven segmentation, in Brain Warping, Academic Press, San Diego, 1999, pp. 67-84. 